\title{
Clinical characteristics of Mycoplasma genitalium and the usefulness \\ of syndromic management among women living with HIV
}

Jason J. Ong, $\mathrm{PhD}^{1,2}$, Mahlape Precious Magooa, $\mathrm{MSc}^{3}$, Admire Chikandiwa, $\mathrm{MD}^{4}$, Helen Kelly, $\mathrm{PhD}^{1}$, Marie-Noelle Didelot, $\mathrm{MD}^{5}$, Etienne E. Muller, $\mathrm{PhD}^{3}$, Vanessa Maseko, BTech ${ }^{3}$, Michel Segondy, $\mathrm{PhD}^{5}$, Sinead Delany-Moretlwe, $\mathrm{PhD}^{4}$, Ranmini Kularatne, FCPath ${ }^{3}$, Philippe Mayaud, MD ${ }^{1}$, on behalf of the HARP Study Group*

${ }^{1}$ Clinical Research Department, Faculty of Infectious and Tropical Diseases, London School of Hygiene \& Tropical Medicine, London, United Kingdom

${ }^{2}$ Central Clinical School, Monash University, Australia

${ }^{3}$ Centre for HIV \& STIs, National Institute for Communicable Diseases, Johannesburg, South Africa

${ }^{4}$ Wits RHI, Faculty of Health Sciences, University of the Witwatersrand, Johannesburg, South Africa

${ }^{5}$ Pathogenesis and Control of Chronic infections, INSERM, University of Montpellier, Montpellier, France

*HARP Study Group composition at end of manuscript

Author for correspondence: Philippe Mayaud, London School of Hygiene \& Tropical Medicine, Keppel Street, London WC1E 7HT, United Kingdom, Telephone: (+44) (0)702 927 2291 Email: philippe.mayaud@lshtm.ac.uk 
Alternate author for correspondence: Jason J. Ong, London School of Hygiene \& Tropical Medicine, Keppel Street, London WC1E 7HT, United Kingdom, Telephone: (+44) (0)7848 698 770

\title{
SHORT SUMMARY
}

A study of women living with HIV (WLHIV) found that Mycoplasma genitalium (MG) is as common ( $\sim 7 \%$ prevalence) as other cervical STIs and syndromic management would miss twothirds of infections.

\begin{abstract}
We report the clinical symptoms and examination findings of Mycoplasma genitalium (MG) in women living with HIV (WLHIV) in South Africa. If we relied on syndromic management alone to treat MG, only 15/46 of MG-infected women would have received appropriate treatment: sensitivity 32.6\% (95\%CI: 19.5-48.0) and specificity 67.4\% (95\%CI: 63.4-71.2).
\end{abstract}

Keywords: Mycoplasma genitalium, sexually transmitted infections (STI), clinical presentation, management, women, HIV 


\section{BACKGROUND}

Mycoplasma genitalium (MG) is a sexually transmitted infection (STI) which may be associated with cervicitis, pelvic inflammatory disease (PID), preterm delivery, infertility, spontaneous abortion and HIV acquisition.(1) MG prevalence in the general population is estimated to be 12\%.(2, 3)

Molecular diagnostic assays for MG are available and have regulatory approval in many countries including Europe, United States, the Middle East, Africa, Australia, New Zealand and parts of Asia. However, due to high costs of such detection methods (for MG as well as for chlamydia and gonorrhoea), few low- and middle-income countries are able to afford STI testing for these pathogens, and instead rely on syndromic management.(4) Though the syndromic approach is pragmatic when expensive diagnostics are not available, it has been criticized for its ineffectiveness in controlling STIs in multiple contexts.(5) Moreover, those infected with an STI but without symptoms or a clear syndrome would not receive treatment.

To date, studies have suggested that HIV-negative women with MG may be more likely to have post-coital bleeding and abnormal vaginal discharge compared to women not infected with MG(2, 6), but detailed symptomatology of MG infection in WLHIV has not been extensively described. This study aims to determine the prevalence and clinical correlates of MG in WLHIV in South Africa and estimate the proportion of missed MG cases by syndromic management. 


\section{METHODS}

\section{Study population}

Cervical specimens used for MG detection were collected as part of an evaluation study of cervical cancer screening strategies in Burkina Faso and South Africa, as previously described (HARP - HPV in Africa Research Partnership).(7) Inclusion criteria were WLHIV, aged 25-50 years, and residing in Ouagadougou or Johannesburg. This analysis focuses on WLHIV recruited in South Africa, as MG prevalence was found to be low in Burkina Faso (4/615, 0.6\%). WLHIV were recruited from primary health care centres and surrounding communities in Johannesburg from December 2011 to October 2012. Data on self-reported clinical symptoms, suspected STI syndromes, socio-demographic and behavioural characteristics were collected by intervieweradministered questionnaire. All participants were examined by trained study nurses and the following examination findings were recorded: vulval and/or vaginal inflammation, vaginal discharge and/or endocervical discharge, cervical contact bleeding, and pelvic pain on bimanual palpation. We also recorded information on the prescription of antibiotics according to the syndromic diagnosis made during the study visit which may be active against MG.

\section{Specimen collection and laboratory analysis}

For all women, a cervical swab was collected to detect Mycoplasma genitalium, Chlamydia trachomatis, Neisseria gonorrhoeae and Trichomonas vaginalis using a validated in-house realtime multiplex polymerase chain reaction (mPCR) assay. Specimens that tested MG positive with the in-house mPCR assay were confirmed with the commercial Sacace MG real-time PCR assay (Sacace Biotechnologies, Como, Italy). All real-time PCR assays were performed using the Rotor-Gene platform (Qiagen, Hilden, Germany). We collected an ecto/endocervical swab using 
Digene cervical sampler (Qiagen, Courtaboeuf, France) for human papillomavirus (HPV) testing (Digene HC-II, Qiagen, Gaithersburg, Maryland USA) and genotyping (INNO-LiPA HPV genotyping Extra assay, Innogenetics, Courtaboeuf, France). A vaginal smear was Gram-stained for the diagnosis of bacterial vaginosis (BV, Nugent's score of 7-10) and Candida spp.

Venous blood was taken to perform herpes simplex virus type 2 (HSV-2) serology (Kalon IgG2 ELISA, Kalon Diagnostics, Guilford, UK), Treponema pallidum haemagglutination assay (TPHA) and Immutrep ${ }^{\circledR}$ rapid plasma reagin (RPR) test (Omega Diagnostics,Scotland, UK), HIV-1 plasma viral load (Cobas Taqman, Roche Diagnostics,New Jersey, USA) and CD4 ${ }^{+} \mathrm{T}-$ lymphocyte count (FACScount, Becton-Dickinson, Franklin Lakes, New Jersey, USA).

\section{Statistical analysis}

Multinomial multivariable logistic regression was used to compare women with no detectable pathogens (i.e. negative for MG and other STIs or reproductive tract infections (RTIs) such as BV and candidiasis) to women with: 1) MG positive, mono-infection; 2) MG positive, coinfected with other STI/RTI; and 3) MG negative but presence of other STI/RTI. We calculated adjusted odds ratios (AORs) alongside 95\% confidence intervals (CI). Sensitivity was calculated as the probability of symptoms among those with MG, and specificity is the fraction of those without MG who do not have symptoms. Data were analyzed using STATA (StataCorp 2013) and we report our findings according to the STROBE statement. 


\section{RESULTS}

In total, 622 women with MG results (out of 623 women recruited) are included in this analysis (Supplementary Table 1). Their mean age was 35 years $( \pm 6.1), 43 \%$ had completed high school or above, and $82 \%$ reported having sex in the last three months.

MG was detected in 7.4\% (46/622, 95\%CI: 5.5-9.7), Trichomonas vaginalis in 16.2\%(95\%CI: 13.4-19.4), Chlamydia trachomatis in 5.0\% (95\%CI: 3.4-7.0), Neisseria gonorrhoeae in 2.3\%(95\%CI: 1.2-3.7), BV in 41.6\%(95\%CI: 37.6-45.6), and Candida spp. in 8.5\%(95\%CI: 6.411.0). MG was a mono-infection in 16 women (2.6\%) and MG with STI/RTI coinfection in 30 women (4.8\%); whilst 343 women (55.1\%) had no MG but another STI/RTI, and 233 women (37.5\%) had no MG and no STI/RTI (uninfected). Symptoms were commonly reported by WLHIV: half of the women $(52 \%, 343 / 623)$ reported at least one of the following symptoms: dysuria, genital itch, lower abdominal pain, vaginal discharge, dyspareunia, or post-coital bleeding.

Among women with MG, half were symptomatic (54\%, 25/46). The most common STI/RTI coinfections were: BV $(47.8 \%, 22 / 46)$, T. vaginalis $(15.2 \%, 7 / 46)$, candidiasis $(10.9 \%, 5 / 46)$, and C. trachomatis $(4.4 \%, 2 / 46)$. In the 16 women with MG mono-infection, the majority were asymptomatic $(75 \%, 12 / 16)$.

Table 1 presents the value of clinical symptoms or examination findings to diagnose MG. We assessed for differences between women who have no STI/RTI (uninfected) compared with women with: 1) MG mono-infection; 2) MG co-infected with another STI/RTI; and 3) no MG 
but have another STI/RTI. We found that it was not possible to distinguish these groups from one another by just using clinical history or examination findings.

If we relied on syndromic management alone (i.e. clinician-suspected PID, vaginal discharge syndrome or any STI syndrome) to treat MG, only 15/46 of MG-infected women would have received any antibiotics: sensitivity 32.6\% (95\%CI: 19.5-48.0) and specificity 67.4\% (95\%CI: 63.4-71.2). One woman received azithromycin and doxycycline, and 14 women received doxycycline alone.

\section{DISCUSSION}

To our knowledge, this is the first study to comprehensively evaluate the clinical profile of MG in WLHIV in a LMIC. We extend the literature by showing MG was as common as other STIs but challenging to diagnose without using molecular diagnostics. The current practice of syndromic STI management will miss two-thirds of MG cases in this population of WLHIV, consistent with another South African study among WLHIV showing that syndromic management would miss 11/16 (69\%) MG.(8) This is higher than missed chlamydia (44\%), gonorrhoea (40\%) and trichomoniasis (33\%) using syndromic management.(8)

The main strength of the study is that we evaluated a large number of WLHIV who had comprehensive assessments of genital symptoms, clinical examination and all were tested for mucosal STI/RTIs. This allowed us to carefully assess the clinical features of MG compared with women with no infection and other STI/RTIs. A limitation with this approach was that the recorded symptoms and clinical findings were dependent on the skills of the trained research 
nurses but this reflects the real-world level of care provided by nurses in South Africa. Our MG prevalence may be an underestimate as we tested cervical swabs (rather than vaginal swabs) and for the purpose of this study, we confirmed all MG positive cases on mPCR with a second MG test (MG Sacace).(9)

The reliance on patient-reported symptoms and clinical signs to identify women with MG would be challenging without molecular diagnostics. MG, by itself, is associated with relatively low inflammation compared to other STIs,(3) and so are often asymptomatic. Our findings that $46 \%$ of women with MG were asymptomatic is similar to a UK-based study that reported $56 \%$ of HIV-negative women with MG were asymptomatic.(2) When we excluded women with other STIs/RTIs, we found that $75 \%$ of women with MG mono-infection were asymptomatic. Though studies in HIV-negative women reported higher likelihood of post-coital bleeding and abnormal vaginal discharge for women with MG,(2, 6) our study among WLHIV demonstrates the difficulties of identifying MG in a real-world setting. The specificity of symptoms or signs for MG was low in a population where half of the women reported at least one urogenital symptom, and a high proportion were diagnosed with bacterial vaginosis (42\%).

Our study findings have direct policy implications. First, the syndromic approach would not be sensitive or specific enough to detect MG in WLHIV. Second, in settings where MG is frequent but no testing is available, the choice of antibiotics included in syndromic STI management should, if possible, treat MG and reflect the local resistance profile of MG to ensure that MG is covered appropriately, and prevent the development of further resistance. Effective management of MG ideally require diagnostics with AMR detection that can also guide therapy.(10) 
In conclusion, MG infection is as common as other cervical STIs among WLHIV in South Africa but is challenging to diagnose based on clinical symptoms and examination findings alone. Molecular diagnostics with AMR testing for MG and Neisseria gonorrhoeae are needed for accurate detection so that appropriate treatment can be offered. In the absence of MG diagnostics, the drugs used in syndromic management of STIs should be carefully selected to cover for MG without fostering the development of resistance. MG-AMR monitoring should be included in future STI-AMR monitoring along that of Neisseria gonorrhoeae. 


\section{Funding}

This work was supported by the European Commission (EC) $7^{\text {th }}$ Framework Programme under grant agreement No. HEALTH-2010-F2-265396.

\section{Conflict of interest}

The authors have no conflicts of interest to disclose.

\section{Acknowledgements}

*HARP Study Group members include: A. Chikandiwa, E. Cutler, S. Delany-Moretlwe, D. A. Lewis, M.P. Magooa, V. Maseko, P. Michelow, B. Muzah, T. Omar, A. Puren (Johannesburg, South Africa); F. Djigma, J. Drabo, O. Goumbri-Lompo, N. Meda, B. Sawadogo, J. Simporé, A. Yonli, S. Zan (Ouagadougou, Burkina Faso); V. Costes, M-N. Didelot, S. Doutre, N. Leventoux, N. Nagot, J. Ngou, M. Segondy (Montpellier, France); and A. Devine, C. Gilham, L. Gibson, H. Kelly, R. Legood, P. Mayaud, H.A. Weiss (London, UK).

We thank the HARP International Scientific Advisory Group (ISAG) constituted of Prof. C. Lacey (Chair, University of York, UK), Prof. Y-L. Qiao (Chinese Academy of Medical Sciences and Peking Union Medical College, Beijing, China), Prof. M. Chirenje (University of Harare, Zimbabwe) and Prof. S. de Sanjosé (Catalan Institute of Oncology, Barcelona, Spain). 


\section{Contributors}

PM, SDM, \& MS conceived and planned the study; AC, SD, HK and PM coordinated the study, participant recruitment and management: MPM, MND and EM performed the lab testing and QC for MG and other STIs, JJO analysed the data and drafted the manuscript, and all authors revised and approved the final manuscript.

\section{Ethical approval}

Ethical approval was granted by the Witwatersrand University in South Africa (no. 110707) and the London School of Hygiene and Tropical Medicine (no. 7400). 


\section{REFERENCES}

1. Lis R, Rowhani-Rahbar A, Manhart LE. Mycoplasma genitalium infection and female reproductive tract disease: a meta-analysis. Clin Infect Dis. 2015;61(3):418-26.

2. Sonnenberg P, Ison CA, Clifton S, et al. Epidemiology of Mycoplasma genitalium in British men and women aged 16-44 years: evidence from the third National Survey of Sexual Attitudes and Lifestyles (Natsal-3). Int J Epidemiol. 2015;44(6):1982-94.

3. Dehon PM, McGowin CL. Mycoplasma genitalium infection is associated with microscopic signs of cervical inflammation in liquid cytology specimens. J Clin Microbiol. 2014;52(7):2398-405.

4. Department of Health, Republic of South Africa. Sexually Transmitted Infections Management Guidelines 2015. Accessed at https://www.health-e.org.za/wpcontent/uploads/2015/06/STIguidelines3-31-15cmyk.pdf. Accessed 10th August 2017.

5. Clark JL, Lescano AG, Konda KA, et al. Syndromic management and STI control in urban Peru. PLoS One. 2009;4(9):e7201.

6. Vandepitte J, Bukenya J, Hughes P, et al. Clinical characteristics associated with Mycoplasma genitalium infection among women at high risk of HIV and other STI in Uganda. Sex Transm Dis. 2012;39(6):487-91.

7. Kelly HA, Sawadogo B, Chikandiwa A, et al. Epidemiology of high-risk human papillomavirus and cervical lesions in African women living with HIV/AIDS: effect of anti-retroviral therapy. AIDS. 2017;31(2):273-85.

8. van der Eem L, Dubbink JH, Struthers HE, et al. Evaluation of syndromic management guidelines for treatment of sexually transmitted infections in South African women. Trop Med Int Health. 2016;21(9):1138-46. 
9. Schachter J, Chow JM, Howard H, Bolan G, Moncada J. Detection of Chlamydia trachomatis by nucleic acid amplification testing: our evaluation suggests that CDCrecommended approaches for confirmatory testing are ill-advised. J Clin Microbiol. 2006;44(7):2512-7.

10. Read TRH, Fairley CK, Murray GL, et al. Outcomes of Resistance-guided Sequential Treatment of Mycoplasma genitalium Infections: A Prospective Evaluation. Clin Infect Dis. 2019;68(4):554-60. 
Table 1. Multinomial multivariable logistic regression to compare among 622 WLHIV in South Africa, those with no MG and no STI/RTI (uninfected,

i.e. MG- STI/RTI-, n=233) with WLHIV who had 1) MG mono-infection (MG+ STI/RTI-, n=16), 2) MG coinfection with another STI/RTI (MG+

STI/RTI+, n=30), and 3) no MG with another STI/RTI (MG- STI/RTI+, n=343)

\begin{tabular}{|c|c|c|c|c|c|c|}
\hline Characteristic & $\begin{array}{r}\text { MG+ STI/RTI- } \\
\text { vs. uninfected } \\
\text { APOR }^{\#}(95 \% \text { CI) }\end{array}$ & P value & $\begin{array}{r}\text { MG+ STI/RTI+ } \\
\text { vs. uninfected } \\
\text { APOR }^{\#}(95 \% \text { CI) }\end{array}$ & P value & $\begin{array}{r}\text { MG- STI/RTI+ } \\
\text { vs. uninfected } \\
\text { APOR }^{\#}(95 \% \text { CI) }\end{array}$ & $P$ value \\
\hline \multicolumn{7}{|l|}{ Self-reported symptoms } \\
\hline Dysuria $(n=80)$ & $0.33(0.04-3.01)$ & 0.325 & $3.23(1.30-8.05)^{*}$ & 0.012 & $1.12(0.66-1.90)$ & 0.663 \\
\hline Genital itch $(n=110)$ & $0.83(0.20-3.42)$ & 0.796 & $1.93(0.79-4.68)$ & 0.146 & $1.16(0.73-1.84)$ & 0.536 \\
\hline Lower abdominal pain (n=158) & $0.23(0.04-1.37)$ & 0.106 & $1.70(0.75-3.86)$ & 0.206 & $1.02(0.69-1.52)$ & 0.911 \\
\hline Vaginal discharge (n=82) & & & $3.77(1.43-9.92)^{* *}$ & 0.007 & $2.07(1.18-3.62)^{*}$ & 0.011 \\
\hline Dyspareunia (n=112) & $0.34(0.04-2.73)$ & 0.312 & $1.55(0.60-3.96)$ & 0.364 & $1.32(0.84-2.07)$ & 0.223 \\
\hline Post coital bleeding ( $\mathrm{n}=35)$ & $0.92(0.10-8.33)$ & 0.940 & $1.03(0.21-5.00)$ & 0.973 & $0.81(0.39-1.69)$ & 0.573 \\
\hline \multicolumn{7}{|l|}{ Clinical signs } \\
\hline Vulval inflammation $(\mathrm{n}=4)$ & $\varnothing$ & - & $7.37(0.41-133.80)$ & 0.177 & $1.23(0.11-13.70)$ & 0.869 \\
\hline Vaginal inflammation $(\mathrm{n}=8)$ & $\varnothing$ & - & $4.55(0.26-78.35)$ & 0.296 & $3.44(0.41-28.98)$ & 0.255 \\
\hline Vaginal discharge $(n=165)$ & $0.41(0.09-1.94)$ & 0.261 & $1.14(0.47-2.77)$ & 0.780 & $1.58(1.06-2.34)^{*}$ & 0.023 \\
\hline Endocervical discharge (n=74) & $0.40(0.05-3.32)$ & 0.399 & $0.47(0.10-2.11)$ & 0.321 & $0.95(0.57-1.60)$ & 0.849 \\
\hline Contact bleeding (n=267) & $0.79(0.26-2.42)$ & 0.682 & $0.82(0.36-1.86)$ & 0.633 & $1.44(1.02-2.03)^{*}$ & 0.038 \\
\hline \multicolumn{7}{|l|}{ Clinician diagnosis } \\
\hline Any STI syndrome (n=192) & $1.03(0.77-1.36)$ & 0.861 & $1.03(0.81-1.31)$ & 0.797 & $1.05(0.86-.1 .28)$ & 0.636 \\
\hline Vaginal discharge syndrome (n=98) & $0.45(0.06-3.58)$ & 0.449 & $2.29(0.91-5.79)$ & 0.079 & $1.49(0.92-2.42)$ & 0.109 \\
\hline Pelvic inflammatory disease (PID) syndrome (n=9) & $\varnothing$ & - & $\varnothing$ & - & $3.00(0.74-12.18)$ & 0.123 \\
\hline
\end{tabular}


${ }^{\varnothing}$ too few cases to estimate model; \# adjusted for age, HIV-1 plasma viral load $>1000$ copies/mL and syphilis; * $\mathrm{p}<0.05,{ }^{* *} \mathrm{p}<0.01$,

*** $\mathrm{p}<0.001 ;$ APOR = adjusted prevalence odds ratio, MG = Mycoplasma genitalium, RTI = reproductive tract infections (i.e. bacterial vaginosis, candidiasis),

PID = pelvic inflammatory disease, STI = sexually transmitted infection (i.e. $N$. gonorrhoeae, C. trachomatis, $T$. vaginalis), $95 \%$ CI $=95 \%$ confidence interval 